\title{
THE IMPROVEMENT OF BRIGHTNESS CORRECTION OF POLYMERIC FOILS IMAGES
}

\author{
RAVAS, R[udolf] \& SYROVA, L[ivia]
}

\begin{abstract}
The contribution treats the topic of analysis of deformations sources of images of visualized transparent polymeric foils by the schlieren methods and afterwards by image processing methods. The brightness deformations are rectifiable by means of schlieren apparatus setting and by the methods of image processing. A new method of correction of brightness distortion is presented. The method is completed by image correction based on the equalizing grayscale local mean values to the global mean value of the image excluding pixels with outlying brightness. The contribution of described correction is illustrated by improving properties of signatures calculated from foils images by the statistical methods of foils classification.
\end{abstract}

Keywords: Schlieren, optical visualization, polymeric foil, brightness correction

\section{INTRODUCTION}

Optical visualization methods utilize changes in the absorption of photons during their transmission through mechanically loaded regions. The most common procedure of visualizing transparent flow field is to record the refractive behavior of flowing medium illuminated by a beam of visible light [1].

With regard to the equipment of our optical laboratory we have chosen schlieren visualization method. Schlieren system serves to measure the amount of light deflection generated by an optical transparent phase object. A parallel light beam traverses the test object and is focused thereafter by means of a lens of spherical mirror, named the schlieren head. Optical apparatus constructed after J. Bolf utilizes small circular diaphragm instead of schlieren head [2]. Light is deviated along the optical path from its nominal course in the absence of refractive - index variations and the amount of light deflection generated by a transparent optical phase can be measured [1]. The apparatus consists of the light source. Light beam traversing the test object is deviated along the optical path from the nominal course corresponding to the absence of variations of refractive index. The camera objective focuses the test object onto the recording plane, where a reduced intensity of light, depending on the amount of light cut off by the carefully adjusted diaphragm, can be observed. Without any disturbances in the optical path the original light source will have uniform reduction in intensity due to the light cut-off by the diaphragm. When there is a disturbance in the optical path, the light rays will be deflected.

Schlieren method has found application in aerodynamics and hydrodynamics of the flowing medium [3], [4]. The biggest advantage is that it provides an illustrative and comprehensive picture of the nature of the flow, e.g. direct resolution of the laminar and turbulent flow modes in optically transparent liquids and combined with implementation of suitable trace particles provides velocity profiles as well. Schlieren method provides useful information on the visually inaccessible objects such as optically transparent materials, especially, polymeric foils [5], [6]. Digital recording can be further processed by means of image processing methods enabling defectoscopy and classification of tested polymeric foils.

\section{BRIGHTNESS CORRECTION}

In order to evaluate images gained by the optical visualization of polymeric foils by image processing it is necessary to correct influence of non-homogeneity of light source, influence of the optical string and the sensing device. One of the possibilities is to use reference image picked up without presence of test object. The reference image can be taken as an image of errors of the whole optical system provided that we have used a virtual light source [6]. Correction of brightness errors is necessary for the further image processing. The brightness disturbances can be caused by non-collinearity of optical axes of the condenser lens and the objective lens [6], non-homogeneity of the light source and impurities in the optical path.

Non-homogeneity of the light source and deformation of the optical path can be successfully described by the multiplicative error model

$$
f(i, j)=f_{I}(i, j) \cdot e(i, j)
$$

where $f(i, j)$ is deformed foil image, $f I(i, j)$ is ideal foil image, $e(i, j)$ is brightness image distortion in pixel with coordinates $\mathrm{i}, \mathrm{j}$. When we scan the image by the schlieren apparatus without presence of the tested foil brightness distortions of the obtained image represent image of distortions of the optical path and non-homogeneity of light source. When we designate such an image as the standard one that is an image of distortions from virtual ideal homogenous light source whose image in ideal case is the image with the constant brightness $\mathrm{c}$

$$
f_{e}(i, j)=e(i, j) . c
$$

Formula for corrected foil image $\mathrm{f}_{\mathrm{C}}(\mathrm{i}, \mathrm{j})$

$$
f_{C}(i, j)=\frac{c}{f_{e}(i, j)} . f(i, j)
$$

The value $\mathrm{c}$ is chosen in order to have brightness value of corrected image in boundaries of display access. 
Correction [6] is based on the fact that in case of the foil without defects each area of image of visualized foil structure of sufficient size should have the same mean value. Image of foil $\mathrm{I}(\mathrm{i}, \mathrm{j})$ of size $\mathrm{NxN}$ has been divided into square disjunctive areas $\mathrm{J}(\mathrm{m}, \mathrm{n})$ of size $\mathrm{KxK}$, where $\mathrm{K}=\mathrm{N} / \mathrm{k}$. Coefficient of correction of radial deformation for the area centre is described by

$$
C\left((m-1) K+\frac{K}{2},(n-1) K+\frac{K}{2}\right)=\frac{\frac{1}{N^{2}} \sum_{i=1}^{N} \sum_{j=1}^{N} I(i, j)}{\frac{1}{K^{2}} \sum_{i=(m-1) K}^{m K} \sum_{j=(n-1) K}^{n K} I(i, j)}
$$

When to put

$$
m_{K}=(m-1) K+\frac{K}{2} \quad n_{K}=(n-1) K+\frac{K}{2}
$$

then the coefficient (6) can be expressed by

$$
C_{m, n}=C\left(m_{K}, n_{K}\right)
$$

We shall calculate value of correction for the arbitrary point of the image by the bilinear transformation of coefficients (7) of four nearest areas centers to the given point. When for $\mathrm{i}, \mathrm{j}$

$$
i \in\left\langle r_{K},(r+1)_{K}\right\rangle, j \in\left\langle s_{K},(s+1)_{K}\right\rangle
$$

and when to mark A, B as

$$
\begin{gathered}
A=C_{r, s}+\frac{C_{r+1, s}-C_{r, s}}{K}\left(i-r_{K}\right) \\
B=C_{r, s+1}+\frac{C_{r+1, s+1}-C_{r, s+1}}{K}\left(i-r_{K}\right)
\end{gathered}
$$

Brightness values of corrected image are calculated from

$$
f_{C R}(i, j)=f_{C}(i, j) \cdot C(i, j)
$$

where

$$
C(i, j)=A+\frac{B-A}{K}\left(j-s_{K}\right)
$$

\section{EXPERIMENTAL RESULTS}

During the experimental analysis we used samples of very thin optically transparent polymeric foils kxe20 (13 samples), kxe30 (6 samples), kxt21 (15 samples), on25 (16 samples) and one12 (11 samples).

For all experiments also represented signature vectors whose components are the arithmetic mean and standard deviation of brightness level of color images green component of foils samples have been used.

In fig. 1 are images of foils samples presented, features values were calculated for images without corrections. As the standard deviation of brightness level is predominantly dependent on the optical path deformation confidence intervals for standard deviation for all sensed samples are almost identical. The average brightness level of images depends on the degree of light absorption through the foil sample and on the adjustment of the light source intensity. The method of brightness correction was applied to the samples images according to (3) with adjusting reference brightness value to 190

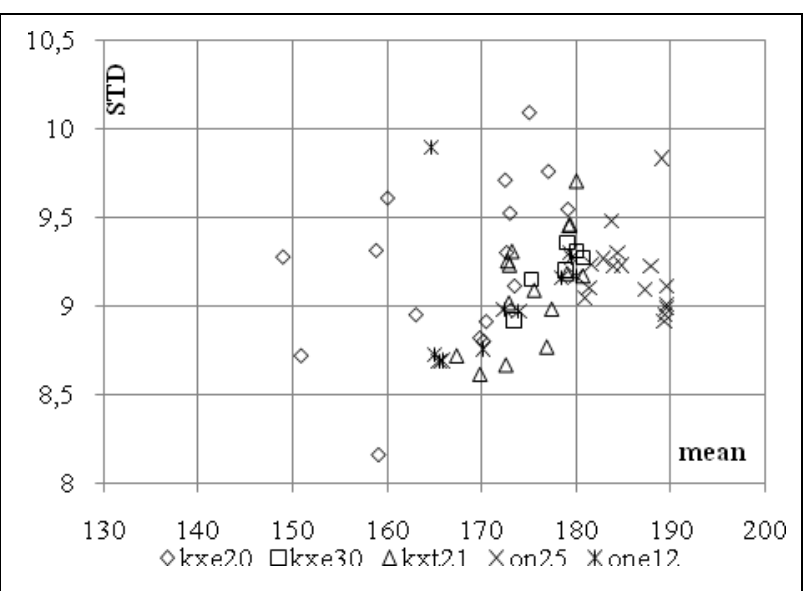

Fig. 1. Patterns of foils images without brightness correction

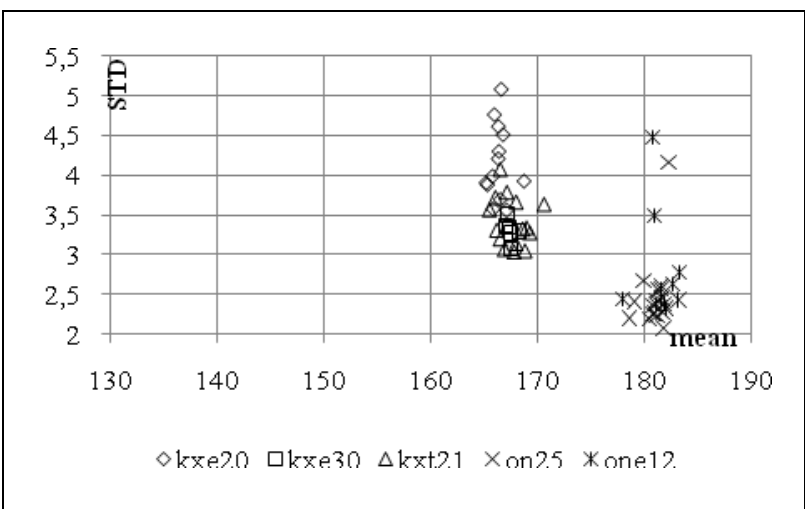

Fig. 2. Patterns of foils images after brightness correction using reference image

In fig. 2 is the tendency of clustering of foils patterns into two groups according to the average values of brightness. Foils samples kxe20, kxe30, kxt21 are characterized by the demonstrably higher light absorption than patterns of other group on 25, one12. There is no clustering of patterns in either of the two groups in the direction of standard deviation values. Insufficient clustering of signatures is caused by several factors.

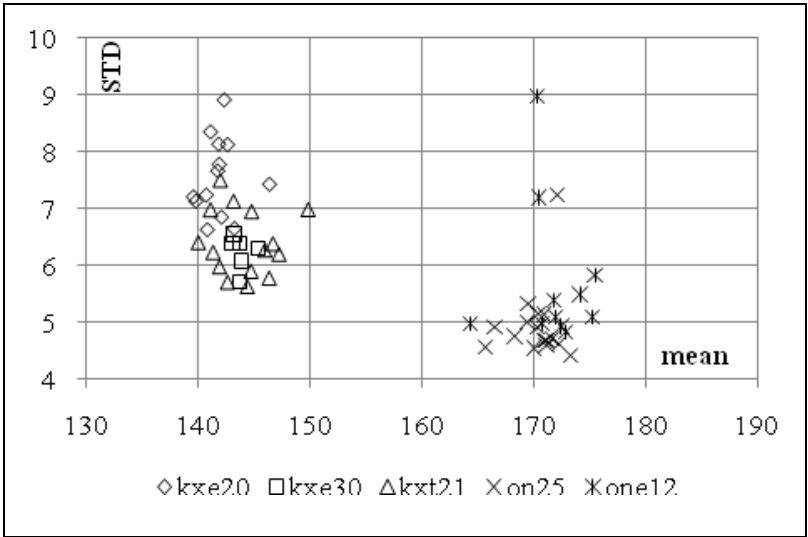

Fig. 3. Patterns of foils images without gamma correction after brightness correction using reference image

Images of visualized foils have been captured by a color still camera because of higher resolution (5 megapixels). Obtained reference images and uncorrected ones are modified by gamma correction, what relationship (3) doesn't consider. In fig. 3 are presented patterns of images of foils samples that before correction according to (3) were transformed into images without 
gamma correction. In this case the value of standard deviation has been increased for all of the images and average brightness levels moved towards lower values.

Quantization error of brightness level with 256 levels of brightness using equation (3) causes that distortion correction towards lower values are characterized by higher relative error. This effect gives rise to the fact that greater brightness distortions are not sufficiently corrected. There is often over-compensation of brightness level.

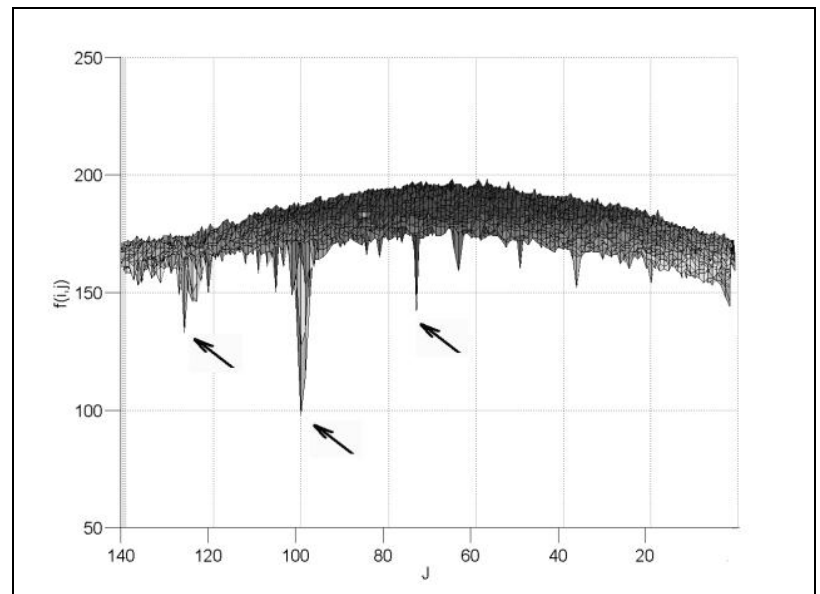

Fig. 4. Projection of 3D-representation of reference image brightness function

Fig. 4 presents brightness profile of the reference image. It can be seen that image brightness distortion are caused by the global tendency of decrement in brightness with increasing distance from the optical axis of the schlieren apparatus. This effect is the dominant factor affecting the value of numerical characteristics based on the differences of brightness levels as the standard deviation. This type of distortion is evident also in the uncorrected image of the foil where it represents dominant component influencing numerical characteristics.

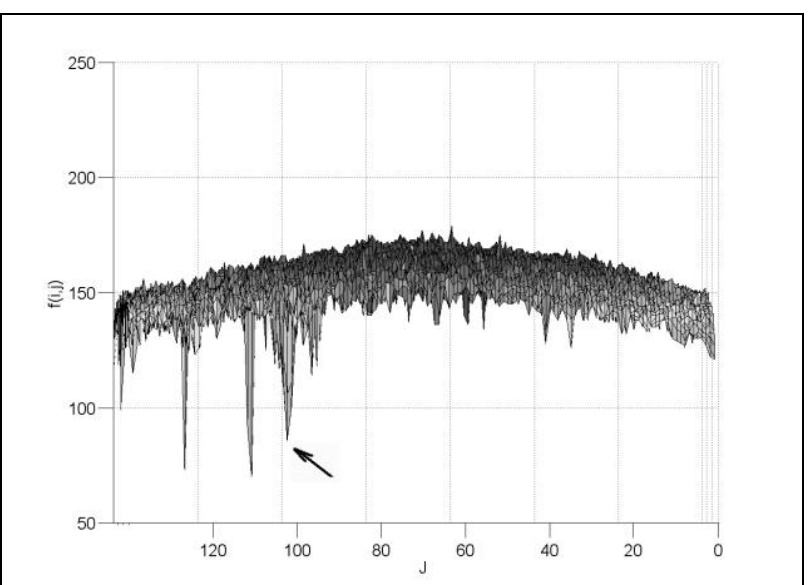

Fig. 5. Projection of 3D-representation of brightness function for noncorrected image of foil KXE20

Another type of distortion is relative large changes of brightness level towards lower values of brightness that are randomly distributed in the image while their position is not affected by the repeating of capture. Their positions in the image are marked by arrows in fig. 4 and fig. 6. Both error components significantly influence properties of non-corrected image of foil sample (fig. 4).
There are further distortions caused by the small defects of the scanned foil (fig. 5) in the case of non-corrected images of very thin foils. The arrow shows the position of such a brightness distortion.

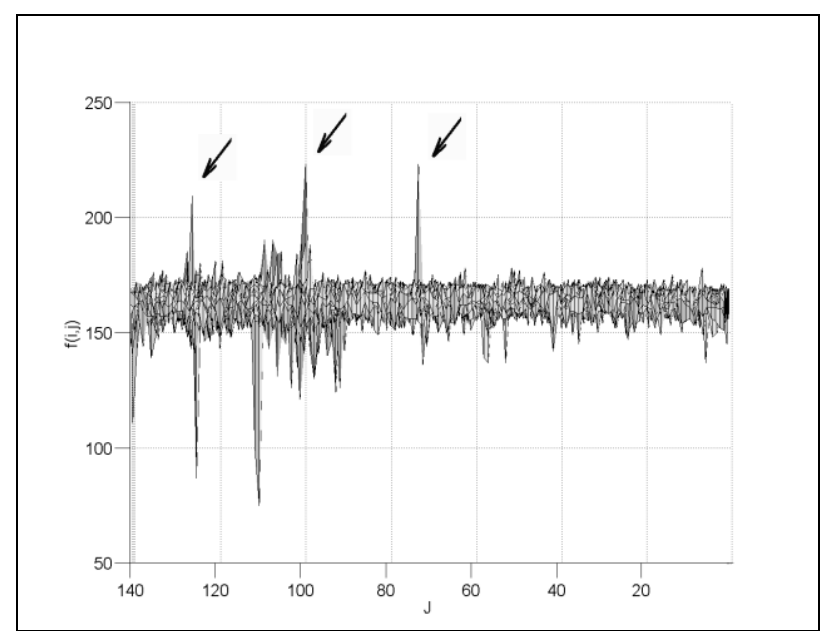

Fig. 6. Projection of 3D-representation of brightness function for corrected image of foil KXE20 corresponding to the formula (3)

Fig. 6 illustrates character of the brightness profile after correction according to (3). Effect of correction of the global tendency of decrement in brightness with increasing distance from the optical axis is suppressed seemingly very well. After application of the method (4) to (8) with parameter step $=50$ pixels to the corrected foils images the variances of brightness levels of corrected images change.

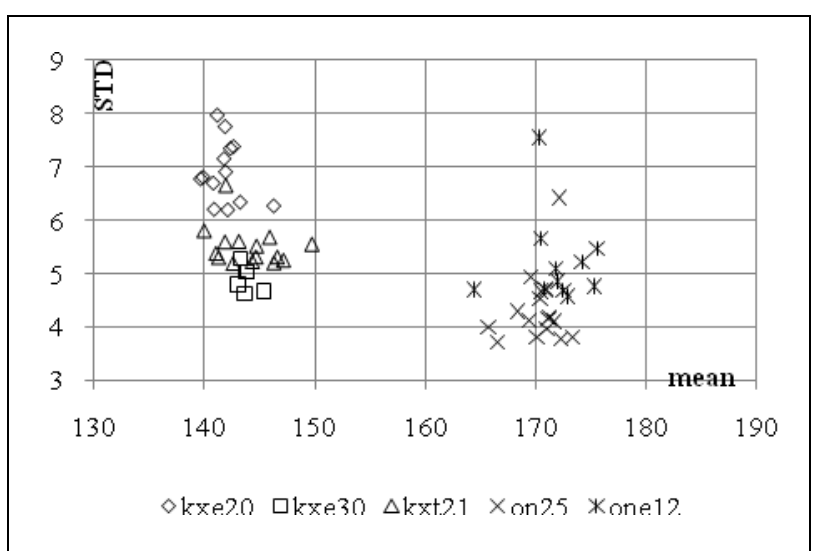

Fig. 7. Patterns of foils images without gamma correction after brightness correction using reference image and consecutive correction using (6) to (8)

Local brightness distortions caused by the imperfection of optical path marked by arrows are in the case of illustration (fig. 6) overcompensated. The presence of brightness distortion caused by the foil damage misrepresents calculation of characteristics from visualized foil image.

\section{PROPOSAL OF THE NEW METHOD}

These facts have led to the proposal of a new method of brightness distortion correction of polymeric foils images. The correction is based on a principle that originally was intended to improve brightness correction of corrected images by the use of reference image. The bottom line is that the correction according to (4) to (8) is applied to uncorrected foils images. Brightness levels of 
uncorrected image are adapted so that average brightness level after correction would by nearby to the average brightness value after correction by reference image according to

$$
f_{1}\left(i_{x} j\right)=\frac{c}{M_{z}} f\left(i_{i} j\right)
$$

where $\mathrm{M}_{\mathrm{e}}$ is mean brightness value of reference image.

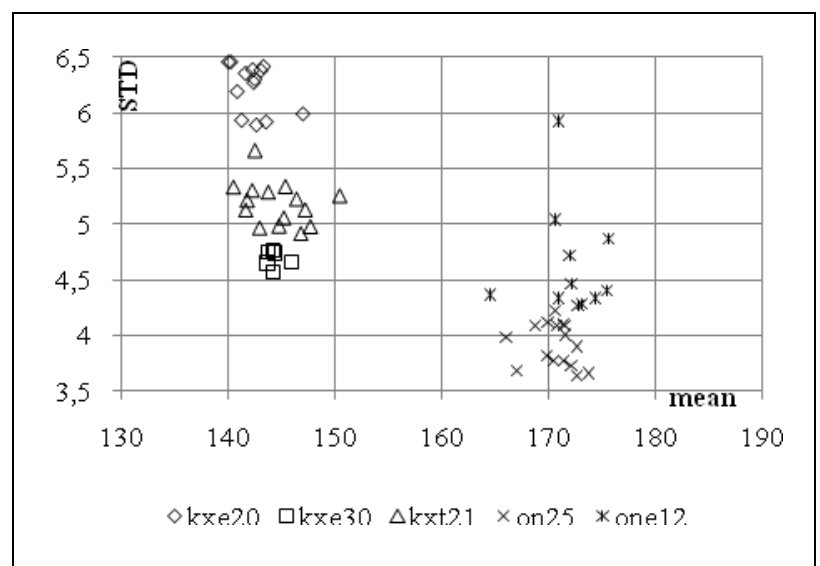

Fig. 8. Patterns of foils images without gamma correction after brightness correction using new method

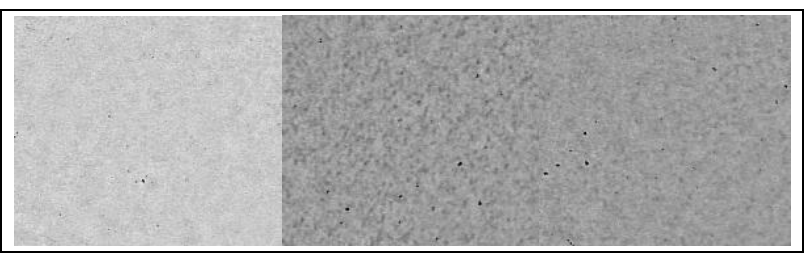

Fig. 9. Example of modified corrected images of visualized foils samples one12, kxe20 and kxt21

Eliminating the impact of local brightness distortions of the optical path and foils damage is based on the exclusion of all pixels from the calculation of characteristics whose brightness level deviation from the average brightness level exceeds the threshold level.

\begin{tabular}{|l|l|l|l|l|}
\hline & & Mean(STD) & MaxSDT & MinSTD \\
\hline \multirow{4}{*}{$\begin{array}{c}\text { Step=50 } \\
\mathrm{T}=3 \sigma\end{array}$} & Kxe20 & 6.224 & 6.456 & 5.885 \\
\cline { 2 - 5 } & Kxt21 & 5.156 & 5.345 & 4.929 \\
\cline { 2 - 5 } & Kxe30 & 4.686 & 4.762 & 4.569 \\
\cline { 2 - 5 } & One12 & 4.636 & 5.927 & 4.267 \\
\cline { 2 - 5 } & On25 & 3.915 & 4.227 & 3.631 \\
\hline \multirow{4}{*}{$\begin{array}{l}\text { Step=50 } \\
\mathrm{T}=2 \sigma\end{array}$} & Kxe20 & 5.609 & 5.820 & 5.279 \\
\cline { 2 - 5 } & Kxt21 & 4.631 & 4.801 & 4.423 \\
\cline { 2 - 5 } & Kxe30 & 4.238 & 4.319 & 4.118 \\
\cline { 2 - 5 } & One12 & 4.178 & 5.120 & 3.873 \\
\cline { 2 - 5 } & On25 & 3.580 & 3.860 & 3.297 \\
\hline \multirow{4}{*}{$\begin{array}{l}\text { Step=20 } \\
\text { T }=3\end{array}$} & Kxe20 & 5.449 & 5.729 & 5.153 \\
\cline { 2 - 5 } & Kxt21 & 4.509 & 4.703 & 4.327 \\
\cline { 2 - 5 } & Kxe30 & 4.137 & 4.198 & 4.040 \\
\cline { 2 - 5 } & One12 & 4.058 & 5.259 & 3.728 \\
\cline { 2 - 5 } & On25 & 3.461 & 3.727 & 3.186 \\
\hline
\end{tabular}

Tab. 1. Mean values, maximum and minimum value of standard deviation of foil images classes for selected step and threshold values $\mathrm{T}$

The threshold is determined as a multiple of standard deviation of the brightness level. Correction of brightness levels excluding outlying brightness levels is performed in two steps, while at the beginning areas in the image with over-threshold deviations of brightness levels are unknown. For the correction it is possible to select values of two parameters, one is the step of correction in algorithm (4) to (9) and second is multiple of standard deviation. The new method of correction with the step 50 pixels excluding outliers beyond $3 \sigma$ from the mean value of brightness of corrected image was applied to samples from fig. 1. From fig. 8 it can be seen that after applying described correction using two signatures it is possible to divide signature space into rectangular sub-spaces that are mutually disjunctive and hereby contain only all images of signature vector belonging to one of mentioned 5 classes. In fig. 9 are images of adjusted sections of corrected selected samples of foils images. Clustering including patterns belonging only to one class of foils is achievable for the other combinations of the step and multiple of standard deviation as well (tab. 1).

\section{CONCLUSION}

The contribution presents new approach to the correction of brightness of visualized polymeric foils images. A new method was verified on the recognition of samples of very thin polymeric foils images. Correction is applicable to images of foils that contain only distortions occupying small area. A jitter of light source of schlieren device is limiting element of brightness correction. In the future, it is necessary to improve the methodology of foils samples capture. In the case of classification of very thin foils by the use of their visualized images it is important to visualize structure in higher resolution, e.g. the use of higher bit depth camera.

\section{ACKNOWLEDGEMENTS}

This work was supported by the Slovak Research and Development Agency under the contract No. APVV0333-11.

\section{REFERENCES}

[1] Smith, A. J. \& Lim, T. T. (2000). Flow Visualization, Imperial College Press, London

[2] Bolf, J.; Bajcsy, J. \& Bolf, P. (1993). Methods of the properties testing of optically transparent materials. Journal of Electrical Engineering, Vol. 44, no. 8, pp. 244-247. (in Slovak)

[3] Bolf, J.; Bajcsy, J.; Syrová, L. \& Kodnár, M. (1995). Visualization of flow through mechanical valvular prothesis, 4th Internat.Workshop on Measurement 95, Smolenice, 29. 21.5.1995, p. 28, Bratislava

[4] Brownlee, C.; Pegoraro, V.; Shankar, S.; McCormick, P.S. \& Hansen, C.D. (2011). Physically-based interactive flow visualization based on schlieren and interferometry experimental techniques, IEEE Transactions on Visualization and Computer Graphics, Vol. 17 , No. 11, pp. $1574-1586$

[5] Castle, D. A.; Gibbins, B. \& Hammer, P. S. (1994). Physica methods for examining and comparing transparent plastic bags and cling films, Journal of Forensic Science Society, Vol. 34, pp. 61-68.

[6] Ravas, R.; Syrova, L. \& Grman, J. (2009). Correction of optical errors in the images of visualized optically transparent polymeric foils, RADIOELEKTRONIKA '09. 19th International Conference, Bratislava, 22-23 April 2009, pp.303 - 306, ISBN: 978-1-42443537-1 